\title{
GROUPS DEFINED BY PERMUTATIONS OF A SINGLE WORD
}

\author{
WILLIAM EMERSON
}

In $[1$, p. 18] a technique of coset enumeration is used to show that the group on two generators $r$ and $s$ which satisfy the two relations

$$
r s^{2}=s^{3} r, \quad s r^{2}=r^{3} s
$$

is in fact the identity group. The question arises as to what relations in a presentation for a group force the group to "collapse" to the identity in general. This problem has been shown to be recursively unsolvable by Rabin [2], but in restricting our attention to a very special class of finitely presented groups generalizing the example above an answer is obtained.

Definition. Let $W=W\left(x_{1}, \cdots, x_{n}\right)$ be a word in the symbols $x_{1}, \cdots, x_{n}$ (not all necessarily explicitly appearing). For $\sigma \in S_{n}$, the symmetric group on $\{1,2, \cdots, n\}, \sigma W$ denotes the word obtained from $W$ by permuting the indices of the $x_{i}$ according to $\sigma: x_{i} \rightarrow x_{\sigma(i)}$. Furthermore for any subset $\beta \subseteq S_{n}$, we define the group

$$
W^{\beta}=\left\{x_{1}, \cdots, x_{n} \mid \sigma W, \sigma \in \mathcal{P}\right\} .
$$

In particular, if $W^{S_{n}}=\{1\}$ we say the word $W$ is symmetrically trivial.

Comment. If $W$ and $V$ are freely equivalent we clearly have $W^{\mathcal{Q}} \cong V^{\mathcal{Q}}$, and hence we need only consider reduced words $W$. Secondly, a word $W$ on $n$ generators may also be formally considered to be on $m$ generators for any $m>n$, in which case $W^{S_{m}}$ and $W^{S_{n}}$ are not generally isomorphic. In any case, the (common) order of the generators $x_{i}$ in $W^{s_{m}}$ divides their (common) order in $W^{S_{n}}$ (since any relation in $W^{S_{n}}$ is also in $W^{S_{m}}$ for $m>n$ ), and hence if $W$ is symmetrically trivial on $n$ generators it is also symmetrically trivial when considered on $m>n$ generators.

The group $W^{S_{n}}$ clearly has the property that any relation among $x_{1}, \cdots, x_{n}$ remains valid after an arbitrary permutation of indices (in particular all $x_{i}$ have the same order), and this principle is utilized in the proof of the next result which gives the explicit structure of the commutator quotient of $W^{s_{n}}$.

THEOREM 1. $W^{S_{n}}$ abelianized is isomorphic to

$$
(Z / d Z)^{n-2} \times(Z / \alpha Z) \times(Z / \beta Z)
$$

Presented to the Society, January 24, 1968; received by the editors June 2, 1968. 
where we set $\alpha_{i}=\sigma_{z_{i}}(W)(1 \leqq i \leqq n)$, the exponent sum of $x_{i}$ in $W$, and

$$
\begin{aligned}
& d=\text { G.C.D. }\left\{\alpha_{i}-\alpha_{j}: 1 \leqq i<j \leqq n\right\}=\text { G.C.D. }\left\{\alpha_{i}-\alpha_{1}: 1<i \leqq n\right\} \\
& \alpha=\text { G.C.D. }\left\{\alpha_{i}: 1 \leqq i \leqq n\right\}=\text { G.C.D. }\left\{\alpha_{1}, d\right\} \\
& \beta=\frac{d}{\alpha}\left|\sum_{1 \leq i \leq n} \alpha_{i}\right| .
\end{aligned}
$$

We use the conventions G.C.D. $\{0\}=0$ and $Z / 0 Z=Z$.

COROLLARY. $W^{S_{n}}$ abelianized is trivial iff

$$
d=1 \text { and } \sum_{1 \leq i \leq n} \alpha_{i}= \pm 1,
$$

and hence these conditions are necessary for $W$ to be symmetrically trivial.

Proof. The corollary is immediate since $d=1 \Rightarrow \alpha=1$. Also, the second equalities in the definitions of $d$ and $\alpha$ follow from the first since $\left(\alpha_{i}-\alpha_{1}\right)-\left(\alpha_{j}-\alpha_{1}\right)=\alpha_{i}-\alpha_{j}$, and we could just have readily used any "fixed" subscript other than 1.

Let $W\left(x_{1}, \cdots, x_{n}\right)=x_{a_{1}}^{e_{1}} x_{a_{2}}^{e_{2}} \cdots x_{a_{k}}^{e_{k}}$. Then $W^{S_{n}}$ abelianized

$$
\begin{aligned}
& =\left\{x_{1}, \cdots, x_{n} \mid x_{i} x_{j} x_{i}^{-1} x_{j}^{-1}(1 \leqq i, j \leqq n), \sigma W\left(\sigma \in S_{n}\right)\right\} \\
(1) & =\left\{x_{1}, \cdots, x_{n} \mid x_{i} x_{j} x_{i}^{-1} x_{j}^{-1}(1 \leqq i, j \leqq m), \sigma\left(\prod_{i} x_{i}^{\alpha_{i}}\right)\left(\sigma \in S_{n}\right)\right\},
\end{aligned}
$$

upon "abelianizing" $W$ and recalling the definition of the $\alpha_{i}$. We now consider the transpositions $\sigma_{i, j} \in S_{n}$ interchanging $i$ and $j$, and note that modulo commutators

$$
\sigma_{i, j}\left(\prod_{i} x_{i}^{\alpha_{i}}\right)=\left(\prod_{i} x_{i}^{\alpha_{i}}\right)\left(x_{i}^{\alpha_{j}-\alpha_{i}} x_{j}^{\alpha_{i}-\alpha_{j}}\right) .
$$

But any permutation $\sigma$ is the product of transpositions, and consequently modulo commutators we have for any $\sigma \in S^{n}: \sigma\left(\prod_{i} x_{i}^{\alpha}\right)$ $=\left(\prod_{i} x_{i}^{\alpha_{i}}\right) T_{1} \cdots T_{k}$ where each $T_{i}$ is of the form $\left(x_{r}^{\alpha_{j}-\alpha_{i}} x_{\xi}^{\alpha_{i}-\alpha_{j}}\right)$ for some choice of $i, j, r, s \in\{1, \cdots, n\}$. Each such $T_{i}$ is a relator in $W^{S_{n}}$ abelianized since $x_{i}^{\alpha_{j}-\alpha_{i}} x_{j}^{\alpha_{i}-\alpha_{j}}$ is by (2) and we may freely permute indices. Now it is easy to see that this collection of relators in $W^{S_{n}}$ abelianized is equivalent to the relations

(3) $\quad x_{i}^{d}=x_{j}^{d}(1 \leqq i<j \leqq n) \quad d=$ G.C.D. $\left\{\alpha_{i}-\alpha_{j}: 1 \leqq i<j \leqq n\right\}$.

Furthermore, $\prod_{i} x_{i}^{\alpha_{i}}=\left(x_{1} \cdots x_{n}\right)^{\alpha_{1}} \prod_{i} x_{i}^{\alpha_{i}-\alpha_{1}}$ implying by (3) that in $W^{S_{n}}$ abelianized 


$$
\prod_{i} x_{i}^{\alpha_{i}}=\left(x_{1} \cdots x_{n}\right)^{\alpha_{1}} x_{1}^{-D} \text { where } D=\sum_{i}\left(\alpha_{1}-\alpha_{i}\right) .
$$

Therefore $W^{S_{n}}$ abelianized

$$
\begin{aligned}
& =\left\{x_{1}, \cdots, x_{n} \mid x_{i} x_{j} x_{i}^{-1} x_{j}^{-1}(1 \leqq i, j \leqq n),\right. \\
& \left.\left(x_{1} \cdots x_{n}\right)^{\alpha_{1}} x_{1}^{-D}, x_{i}^{d} x_{j}^{-d} \quad(1 \leqq i<j \leqq n)\right\},
\end{aligned}
$$

that is a set of defining relations for $W^{S_{n}}$ abelianized (in addition to the abelian condition) is

(5) (i) $x_{i}^{d}=x_{j}^{d}, 1 \leqq i<j \leqq n$,

(ii) $\left(x_{1} \cdots x_{n}\right)^{\alpha_{1}}=x_{1}^{D}$.

Now define $\alpha=$ G.C.D. $\left\{\alpha_{1}, d\right\}=$ G.C.D. $\left\{\alpha_{i}: 1 \leqq i \leqq n\right\}$, and let $\alpha=r \alpha_{1}+s d, r, s, \in Z$. From (i) we obtain

(iii) $\left(x_{1} \cdots x_{n}\right)^{d}=x_{1}^{n d}$, and from (ii) and (iii),

(6) (iv) $\left(x_{1} \cdots x_{n}\right)^{\alpha}=x_{1}^{F}$ where $F=r D+s n d$,

and $d \mid F$ since $d \mid D$. Since $\alpha \mid \alpha_{1}$ (iv) implies $\left(x_{1} \cdots x_{n}\right)^{\alpha_{1}}=x_{1}^{P \alpha_{1} / \alpha}$, and thus from (ii) $x_{1}^{D}=x_{1}^{F \alpha_{1} / \alpha}$. Hence we may replace (5) by

(7) (i) $x_{i}^{d}=x_{1}^{d}, 1<i \leqq n$,

(ii) $\left(x_{1} \cdots x_{n}\right)^{\alpha}=x_{1}^{F}$,

(iii) $x_{1}^{D}=x_{1}^{F \alpha_{1} / \alpha}$.

Now consider these relations with respect to the new generators

$$
\begin{aligned}
& y_{i}=x_{1} \cdots x_{i} x_{1}^{-i}, \quad 1 \leqq i<n, \\
& y_{n}=x_{1} \cdots x_{n} x_{1}^{-F / \alpha} .
\end{aligned}
$$

They become

(i) ${ }_{y_{i}^{d}}^{d}=1, \quad 1<i<n, \quad y_{1}^{(F d / \alpha)-n d}=1$,

(ii) $y_{n}^{\alpha}=1$,

(iii) $y_{1}^{\left(F \alpha_{1} / \alpha\right)-D}=1$.

Hence we see that $W^{s_{n}}$ abelianized is the direct product of the cyclic groups generated by $y_{1}, \cdots, y_{n}$, and all that remains is the explicit computation of $\beta$, the order of $y_{1}$. From (i) and (iii) we have

$$
\beta=\text { G.C.D. }\left\{F \alpha_{1} / \alpha-D, F d / \alpha-n d\right\},
$$

and

$$
\begin{gathered}
\frac{\alpha_{1}}{\alpha}\left(F \frac{d}{\alpha}-n d\right)-\frac{d}{\alpha}\left(F \frac{\alpha_{1}}{\alpha}-D\right)=\frac{d}{\alpha}\left(D-n \alpha_{1}\right) \\
\Rightarrow \beta \mid \frac{d}{\alpha} \sum_{i} \alpha_{i}, \text { by }(4) .
\end{gathered}
$$


on the other hand, we conclude by verifying that

$$
\frac{d}{\alpha}\left(n \alpha_{1}-D\right)=\frac{d}{\alpha} \sum_{i} \alpha_{i}
$$

divides both $(F d / \alpha)-n d$ and $\left(F \alpha_{1} / \alpha\right)-D \Rightarrow \beta=(d / \alpha)\left|\sum_{i} \alpha_{i}\right|$. From (6), $\alpha=r \alpha_{1}+s d, F=r D+s n d$ implying

$$
\begin{aligned}
(F d / \alpha)- & n d \\
& =(d / \alpha) r D+(d / \alpha) S n d-n d \\
& =(d / \alpha) r D+(d / \alpha) n\left(\alpha-r \alpha_{1}\right)-n d=-r\left((d / \alpha)\left(n \alpha_{1}-D\right)\right) \\
\left(F \alpha_{1} / \alpha\right)- & D \\
& =\left(\alpha_{1} / \alpha\right) r D+\left(\alpha_{1} / \alpha\right) s n d-D \\
& =(D / \alpha)(\alpha-s d)+\left(\alpha_{1} / \alpha\right) s n d-D=s\left((d / \alpha)\left(n \alpha_{1}-D\right)\right)
\end{aligned}
$$

We now consider words $W$ in two generators, in which case a necessary condition for the symmetric triviality of $W$ is $\left\{\left|\alpha_{1}\right|,\left|\alpha_{2}\right|\right\}$ $=\{0,1\}$ by the corollary. It is too much to hope that this condition is also necessary in general since it only depends on the exponential sums of the generators in $W$. Nevertheless, we have the following result which generalizes the example of [1]:

THEOREM 2. If $W(x, y)$ is any word in $x$ and $y$ of syllable length less than six, $W$ is symmetrically trivial $\Leftrightarrow\left\{\left|\alpha_{1}\right|,\left|\alpha_{2}\right|\right\}=\{0,1\}$.

Proof. Without loss of generality we may assume $W$ is cyclically reduced, and also (interchanging $x$ and $y$ and/or replacing $W$ by $W^{-1}$ if necessary) that

$$
W(x, y)=x^{-(a+c)} y^{b} x^{a} y^{-b}
$$

where $\epsilon=+1$ or $-1, a \in N$ and $b \in Z$. Hence the two basic defining relations are

(I) $y^{b} x^{a} y^{-b}=x^{a+e}$,

(II) $x^{b} y^{a} x^{-b}=y^{a+c}$.

Relation (II) implies

$$
x^{b} y^{a b} x^{-b}=y^{(a+\epsilon) b}
$$

while (I) implies

$$
y^{b} x^{a^{2}} y^{-b}=x^{(a+\epsilon) a}, \quad y^{2 b} x^{a^{2}} y^{-2 b}=x^{(a+\epsilon)^{2}},
$$

and by induction on $n$

$$
y^{n b} x^{a^{n}} y^{-n b}=x^{(a+\epsilon)^{n}} \quad(n \in N) .
$$


Setting $n=a+\epsilon$ in (10) we obtain

$$
y^{(a+c) b} x^{a(a+c)}=x^{(a+c)^{a+c}} y^{(a+c) b}
$$

which with (9) implies

$$
\left(x^{b} y^{a b} x^{-b}\right) x^{a^{a+c}}=x^{(a+c)^{a+c}}\left(x^{b} y^{a b} x^{-b}\right) \Rightarrow y^{a b} x^{(a+c)}=x^{(a+c)^{a+c} y^{a b}}
$$

or

$$
y^{a b} x^{a(a+e)} y^{-a b}=x^{(a+c)^{a+c}} .
$$

Setting $n=a$ in (10) we obtain

$$
y^{a b} x^{a} y^{-a b}=x^{(a+c)^{a}}
$$

We consider two cases:

If $\epsilon=+1$ we obtain from (12)

$$
y^{a b} x^{a(a+\epsilon)} y^{-a b}=x^{a(a+\epsilon)^{a}},
$$

while if $\epsilon=-1$ we obtain from (11)

$$
\left.y^{a b} x^{a} y^{-a b}=x^{a(a+c)}\right)^{a+e} .
$$

Consequently, if $\epsilon=+1(11)$ and $(12)^{\prime} \Rightarrow$

$$
x^{(a+\epsilon)^{a}}=1 \text {, }
$$

and if $\epsilon=-1(11)^{\prime}$ and $(12) \Rightarrow x^{(a+c)^{a}}=x^{a(a+c)^{a+e}}, x^{(a+e)^{a+e}}=1, \Rightarrow$

$$
x^{(a+c)^{a}}=1 \text {. }
$$

In either case, using (13) in conjunction with (12) we obtain

$$
x^{a^{a}}=1,
$$

which, since $\left(a^{a},(a+\epsilon)^{a+e}\right)=1$, implies $x=1$, and hence by symmetry $y=1$ also.

\section{REFERENCES}

1. H. S. M. Coxeter and W. O. Moser, 2nd ed., Generators and relations for discrete groups, Springer-Verlag, New York, 1965.

2. M. O. Rabin, Recursive unsolvability of group theoretic problems, Ann. of Math. (2) 67 (1958), 172-194.

Courant Institute of Mathematical Sciences, New York University 